Supporting Information for

\title{
Silica Surface Charge Enhancement at Elevated Temperatures Revealed by Interfacial Water Signals
}

\author{
Md. Shafiul Azam, ${ }^{1}$ Canyu Cai, ${ }^{1}$ Julianne M. Gibbs, ${ }^{2}$, Eric Tyrode, ${ }^{3}$ Dennis K. Hore ${ }^{1,4}$ \\ ${ }^{1}$ Department of Chemistry, University of Victoria, Victoria, British Columbia, V8W 3V6, Canada \\ ${ }^{2}$ Department of Chemistry, University of Alberta, Edmonton, Alberta, T6G 2G2, Canada \\ ${ }^{3}$ Department of Chemistry, KTH Royal Institute of Technology, Stockholm SE-10044, Sweden \\ ${ }^{4}$ Department of Computer Science, University of Victoria, Victoria, British Columbia, V8W 3P6, Canada
}

\section{S1 Experimental details}

A detailed description of the laser system used for our vibrational SFG spectroscopy experiments has been described previously. ${ }^{1}$ Briefly, the system consists of a Nd:YAG laser (Ekspla PL2241A) that generates a 30 ps pulse with a repetition rate of $10 \mathrm{~Hz}$ at $1064 \mathrm{~nm}$ (fundamental) and $532 \mathrm{~nm}$ (second harmonic). A portion of the second harmonic output and the fundamental are used to pump an optical parametric generator (Ekspla PG501) to produce tunable IR frequencies from $2800-3700 \mathrm{~cm}^{-1}$. The IR beam $(\approx 230 \mu \mathrm{J} /$ pulse $)$ and second harmonic visible output $(120 \mu \mathrm{J} /$ pulse $)$ were then transmitted through polarizers, and finally incident on the surface of an IR-grade fused silica dove prism (Del Mar Photonics), approaching at $\theta_{\mathrm{IR}}=74.3 \pm 0.3^{\circ}$ and $\theta_{\mathrm{vis}}=64.5 \pm 0.3^{\circ}$. Just before the surface, the each beam has a diameter of $d=600 \pm 100 \mu \mathrm{m}$. Since we are using such large angles, the intensity is significantly reduced as the area of the beams at the surface is $\frac{1}{4} \pi d^{2} / \cos \theta$. The prism was mounted against a custom Teflon vessel with a fluoropolymer O-ring (Marco Rubber, NH) and the vessel was filled with $18.2 \mathrm{M} \Omega \cdot \mathrm{cm}$ deionized water (Milli-Q). The temperature of the water was varied by employing a cooling and heating circulator (PolyScience 9112) channelled through an aluminum block surrounding the Teflon cell. A thermometer was immersed in the Teflon cell to read the actual temperature of water in contact with silica surface. The SFG light generated from the silica-water interface was then collected at $20 \mathrm{~cm}^{-1}$ IR wavenumber steps and average of 100 laser shots were recorded.

The prism was cleaned before each experiment by dipping into a $500 \mathrm{~mL}$ glass beaker containing piranha solution (3:1 mixture of sulfuric acid and 30\% hydrogen peroxide) for $1 \mathrm{~h}$. (Note: Use caution, piranha solution reacts explosively with organic compounds.) The prism was then taken out of the beaker, rinsed copiously in $18.2 \mathrm{M} \Omega \cdot \mathrm{cm}$ deoinized water $(5 \times 300 \mathrm{~mL})$, sonicated in $300 \mathrm{~mL}$ water for $2 \mathrm{~min}$, and again rinsed with water $(2 \times$ $300 \mathrm{~mL}$ ) to completely remove any acid. The piranha-cleaned prism was then placed in a preheated oven at $85^{\circ} \mathrm{C}$ for $1 \mathrm{~h}$ under vacuum. 


\section{S2 Background}

For charged aqueous surfaces, the water response is considered to have a surface contribution through $\chi^{(2)}$ and a bulk contribution through $\chi^{(3)}$. The pioneering work of Eisenthal et al. has recognized that the $\chi^{(3)}$ contribution is modulated by the surface potential $\Phi_{0}$ and proposed that $I \propto\left|\chi^{(2)}+\Phi_{0} \chi^{(3)}\right|^{2}{ }^{2}$. It was later realized that the full surface potential is not experienced at all ionic strengths, and that an angle- and ionic strength-dependent factor $f_{3}$ should be included so that $I \propto\left|\chi^{(2)}+f_{3} \Phi_{0} \chi^{(3)}\right|^{2}$. $^{3,4}$ It has recently been demonstrated that, at low ionic strengths where the surface potential is high, the Debye-Hückle approximation is not valid, and the product $f_{3} \Phi_{0}$ should be replaced by a more accurate function of the surface potential that we call $g_{3}$ resulting in ${ }^{5}$

$$
I \propto|L L L|^{2}\left|\chi^{(2)}+g_{3} \chi^{(3)}\right|^{2}
$$

where

$$
g_{3}=\Phi_{0}-\frac{4 i k T \Delta k}{e} \sum_{n=1}^{\infty} \frac{\xi^{2 n-1}}{(2 n-1)[i \Delta k-\kappa(2 n-1)]}
$$

and

$$
\xi=\tanh \left[\frac{e \Phi_{0}}{4 k T}\right] .
$$

Here $e$ is the electron charge, $k$ is Boltzmann's constant, $T$ is the absolute temperature, and $L$ are the local field corrections that are described in more detail below. These expressions also account for the angle-dependence of the SFG intensity. ${ }^{5,6}$ Fig. S1 illustrates the variation in the magnitude and phase of $g_{3}$ over the temperature range of interest. The ratio of $\left|\chi^{(2)}\right| /\left|\chi^{(3)}\right|$ against temperature has been plotted in Fig. S2.

In the following sections, we describe the temperature-dependence of the remaining terms in Eq. S1.

\section{S3 Dielectric constant, refractive index, and local fields}

As the model presented above requires consideration of the local field correction factors $L$, we need to account for the effect of temperature on the linear optical properties. The temperature-dependent dielectric constant of water $\left(\varepsilon_{\mathrm{R}}\right)$ can be represented by the linearresponse formula ${ }^{7}$

$$
\varepsilon_{\mathrm{R}}=1+\frac{4 \pi\left|\mathbf{M}^{\mathrm{w}}\right|^{2}}{V k_{\mathrm{B}} T}
$$

where $V$ is the volume of the simulation cell and $\mathbf{M}^{\mathrm{w}}$ is the total dipole moment of the water molecules obtained in an MD simulation by Joutsuka et al. ${ }^{8}$ We obtain the temperaturedependence of $\varepsilon_{\mathrm{R}}$ by interpolating their simulation data. The final expression for the temperature-dependent dielectric constant of water is

$$
\varepsilon_{\mathrm{R}}=1+\frac{22415.8721}{T}
$$

Wavelength- and temperature-dependent refractive index of water were taken from the 

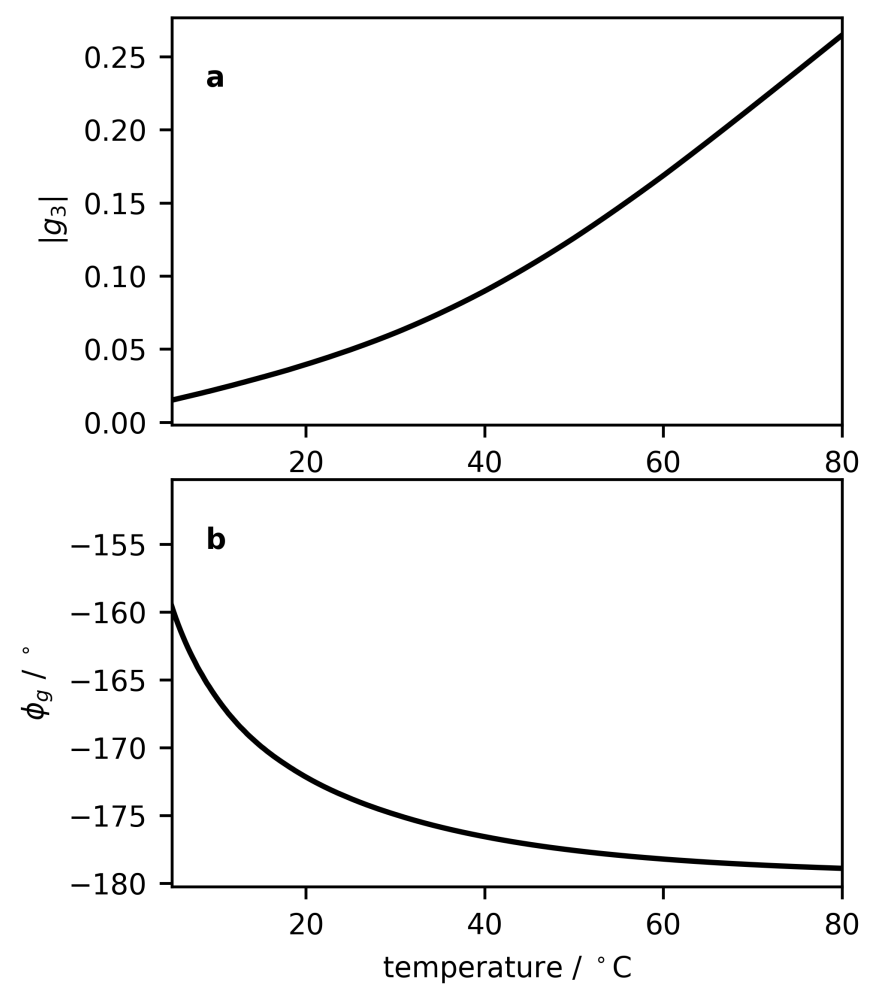

Figure S1: (a) Magnitude and (b) phase of $g_{3}$ as a function of temperature.

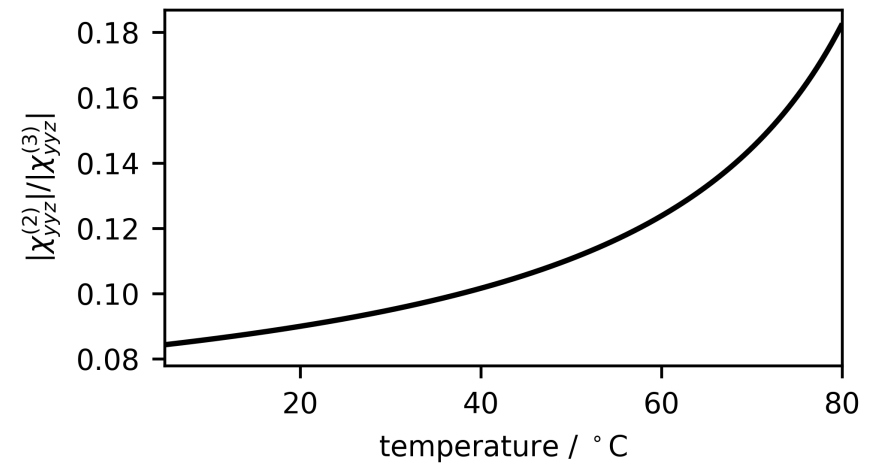

Figure S2: The ratio of $\left|\chi^{(2)}\right| /\left|\chi^{(3)}\right|$ as a function of temperature. 


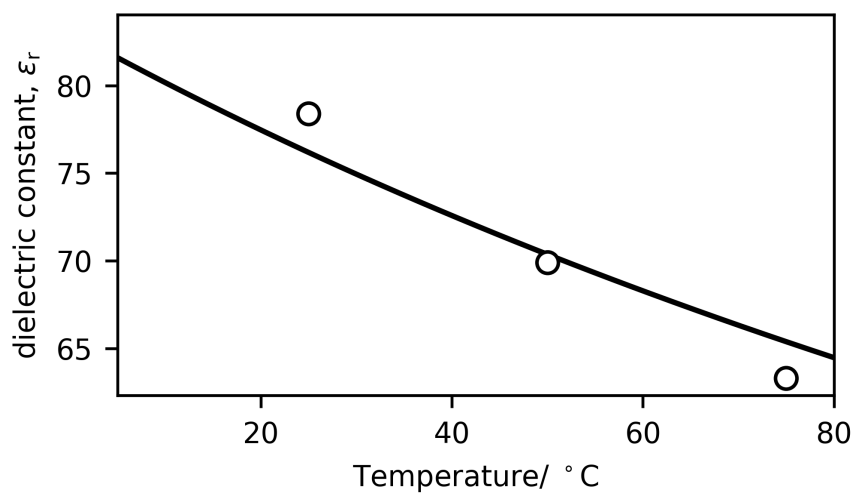

Figure S3: Fitted curve of temperature-dependent dielectric constant of water and experimental data from Joutsuka et al. ${ }^{8}$

literature using 9

$$
n(\lambda, t)=A(t)+\frac{B(t)}{\lambda^{2}}+\frac{C(t)}{\lambda^{4}}+\frac{D(t)}{\lambda^{6}}
$$

where $A, B, C$, and $D$ are the Cauchy coefficients as a function of temperature ( $t$ in Celcius)

$$
\begin{aligned}
& A(t)=1.3208-1.2325 \times 10^{-5} t-1.8674 \times 10^{-6} t^{2}+5.0233 \times 10^{-9} t^{3} \\
& B(t)=5208.2413-0.5179 t-2.284 \times 10^{-2} t^{2}+6.9608 \times 10^{-5} t^{3} \\
& C(t)=-2.5551 \times 10^{8}-18341.336 t-917.2319 t^{2}+2.7729 t^{3} \\
& D(t)=9.3495+1.7855 \times 10^{-3} t+3.6733 \times 10^{-5} t^{2}-1.2932 \times 10^{-7} t^{3} .
\end{aligned}
$$

The temperature-dependent refractive index of silica was also incorporated in our model using the literature expression ${ }^{10}$

$$
n^{2}-1=\sum_{i=1}^{3} \frac{a_{i}}{b_{i}^{2}-E^{2}}
$$

and,

$$
\begin{aligned}
& a_{i}=a_{i 0}+a_{i 1} t+a_{i 2} t^{2} \\
& b_{i}=b_{i 0}+b_{i 1} t+b_{i 2} t^{2} .
\end{aligned}
$$

$E$ is the photon energy in electron volt, and $t$ is the temperature in Celsius. Values of the parameters $a_{\text {in }}$ and $b_{\text {in }}$ can be found in the literature. ${ }^{10}$

The local field coefficients were then calculated using the temperature-dependent refractive index of water and fused silica

$$
(L L L)_{y y z}=L_{y}^{\mathrm{SFG}} L_{y}^{\mathrm{vis}} L_{z}^{\mathrm{IR}}
$$

with

$$
\begin{aligned}
& L_{y}=1+r_{s} \\
& L_{z}=\left(1+r_{p}\right) \sin \left(\theta_{1}\right)\left(\frac{n_{1}}{n^{\prime}}\right)^{2}
\end{aligned}
$$


where

$$
\begin{aligned}
r_{s} & =\frac{n_{1} \cos \left(\theta_{1}\right)-n_{2} \cos \left(\theta_{2}\right)}{n_{1} \cos \left(\theta_{1}\right)+n_{2} \cos \left(\theta_{2}\right)} \\
r_{p} & =\frac{n_{2} \cos \left(\theta_{1}\right)-n_{1} \cos \left(\theta_{2}\right)}{n_{1} \cos \left(\theta_{2}\right)+n_{2} \cos \left(\theta_{1}\right)} \\
n^{\prime} & =\frac{n_{1}+n_{2}}{2} .
\end{aligned}
$$

In the above expressions, $n_{1}$ is the refractive index at the appropriate wavelength and temperature in fused silica and $n_{2}$ is the refractive index of corresponding wavelength and temperature in water. $\theta_{1}$ and $\theta_{2}$ are the incident angle and refracted angle at the corresponding wavelength.

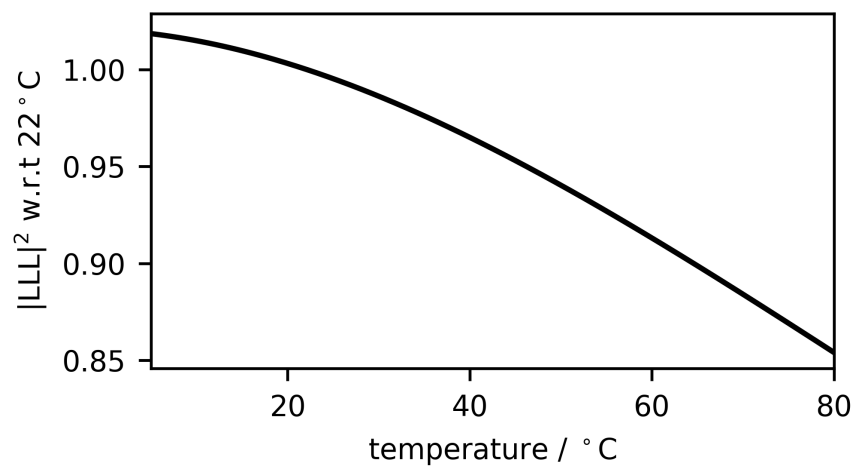

Figure S4: Magnitude squared of the product of the three local field coefficients, $|L L L|^{2}$, normalized to $22^{\circ} \mathrm{C}$.

\section{S4 Deprotonation model}

Our model of two different silanol sites is described by a $\mathrm{p} K_{a}$ for each site, with a more acidic silanol group at $\mathrm{p} K_{a}=5.7$ and a less acidic silanol at $\mathrm{p} K_{a}=8.5$. ${ }^{11}$ We assume that the deprotonation of each site is described by an equilibrium constant

$$
K_{a}=\frac{\left[\mathrm{H}^{+}\right]_{0}\left[\mathrm{SiO}^{-}\right]_{0}}{[\mathrm{SiOH}]_{0}}
$$

where the surface proton concentration $\left[\mathrm{H}^{+}\right]_{0}$ can be expressed in terms of that in the bulk $\left[\mathrm{H}^{+}\right]_{\infty}$ by

$$
\left[\mathrm{H}^{+}\right]_{0}=\left[\mathrm{H}^{+}\right]_{\infty} \exp \left[-\frac{e \Phi_{0}}{k T}\right] .
$$

The temperature-dependent $\mathrm{pH}$ data of pure water were taken from Truman. ${ }^{12}$ This enables us to write the equilibrium constant in terms of the fraction of the surface that is deprotonated, $\alpha$

$$
K_{a}=\frac{\alpha}{1-\alpha}\left[\mathrm{H}^{+}\right]_{\infty} \exp \left[-\frac{e \Phi_{0}}{k T}\right]
$$


Even though we cannot rearrange Eq. S14 to obtain an analytical expression for $\alpha$, the equation can be solved numerically from

$$
\sqrt{I}=\frac{\alpha e}{A_{\mathrm{M}} \sqrt{8 \varepsilon_{\mathrm{R}} \varepsilon_{0} k T}}\left[\sinh \left(\frac{1}{2}\left(\ln \left[\mathrm{H}^{+}\right]_{\infty}-\ln K_{a}-\ln \left(\frac{1-\alpha}{\alpha}\right)\right)\right)\right]^{-1} .
$$

We therefore obtain the degree of deprotonation for each of the sites $\alpha_{1}$ ( $\mathrm{p} K_{a}=5.7$, green curve in Fig. $4 \mathrm{~b})$ and $\alpha_{2}\left(\mathrm{p} K_{a}=8.5\right.$, red curve in Fig. $\left.4 \mathrm{~b}\right)$.

\section{S5 Surface charge density and surface potential}

Now that we have a model for $\alpha_{1}$ and $\alpha_{2}$, we can calculate the surface charge density from the surface area per silanol, based on the relative population of each site. The surface charge density $\sigma$ is given by

$$
\sigma=\frac{\alpha_{1} e}{A_{1}}+\frac{\alpha_{2} e}{A_{2}}
$$

where $A$ is the area per silanol $A_{\mathrm{M}}$, and $e$ is the electron charge. $A$ needs to take the relative population of the two sites into account. Early work considered the two sites to have population ratios of 19:81 at high ionic strength. ${ }^{2}$ We model a ratio of 40:60 based on experiments performed at low ionic strength. ${ }^{13,14}$ Using an area per silanol group of $90 \AA^{215,16}$ we arrive at $A_{1}=227 \AA^{2}$ and $A_{2}=152 \AA^{2}$. This then enables calculation of the surface potential through

$$
\Phi_{0}=\frac{2 k T}{e} \sinh ^{-1}\left[\frac{\sigma}{\sqrt{8 I \varepsilon_{\mathrm{R}} \varepsilon_{0} k T}}\right] .
$$

The resulting surface potential of the silica-water interface with change of temperature is plotted in Fig. S5.

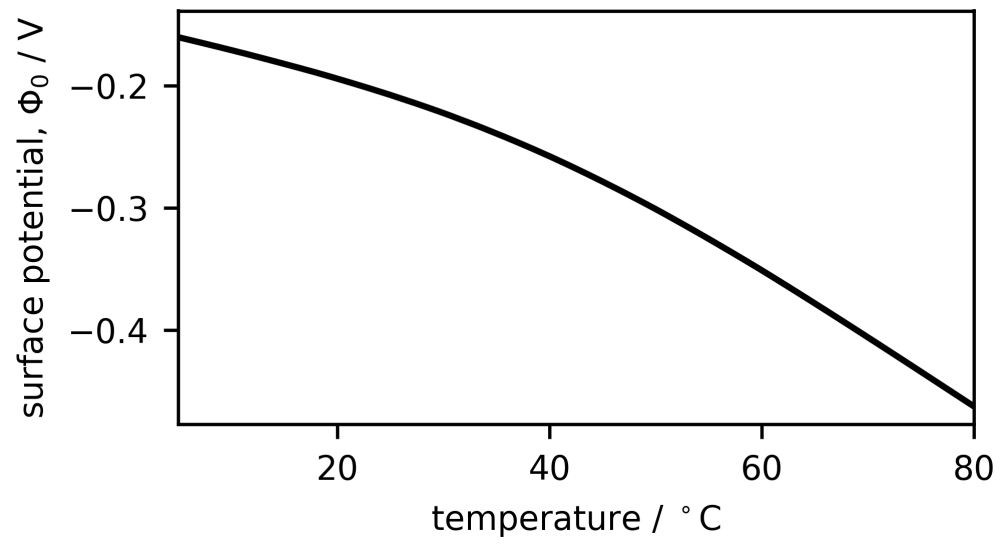

Figure S5: Calculated surface potential using a combination of two different silanol sites. 


\section{S6 Data fitting procedure}

The van't Hoff equation was used to describe the $\ln K_{a}$ change with temperature.

$$
\ln K_{a}=-\frac{\Delta H}{R T}+\frac{\Delta S}{R}
$$

The ultimate goal is to find the $\Delta S$ and $\Delta H$ for the two silanol species that account for the trend in the SFG data. Since we are using literature $\mathrm{p} K_{a}$ values at $22^{\circ} \mathrm{C}$ for two species, the relationship between $\Delta S$ and $\Delta H$ can be expressed as

$$
\Delta S=R\left[\frac{\Delta H}{295.15 \mathrm{~K}}+\log _{e}\left(10^{-\mathrm{p} K_{a}}\right)\right]
$$

for each species. We constructed a function that takes the $\Delta H$ values and evaluates the residual between the predicted SFG signal using our model described above and the experimental data. We then performed an exhaustive search spanning from 0-1000 kJ/mol for $\Delta H$ of both silanol species $\left(\Delta H_{1}\right.$ and $\left.\Delta H_{2}\right)$. After our code finds the global minimum, it further optimizes the result with a downhill simplex algorithm.

\section{S7 Observations in other polarization schemes}

Our model considers that there is no significant reorientation for surface-bound water molecules when the temperature increases. We therefore consider that the temperaturedependence of $\chi^{(2)}$ follows solely from the temperature-dependence of the linear optical properties that comprise the hyperpolarizability in the molecular frame. ${ }^{17}$ We have chosen to use the SSP results in the modelling, as more quantitative treatment requiring local field correction of the SSP data is possible on account of the single $\chi^{(2)}$ tensor element $\left(\chi_{y y z}^{(2)}\right)$ that contributes to SSP signals. To further investigate this point, we have performed measurements in the PPP polarization scheme (all beams p-polarized). As seen in Fig. S6, the PPP trend is very similar to our SSP results. This indicates that there is no significant reorientation of water molecules with temperature, validating our premise that $\chi^{(3)}$ is a more significant contributor to the intensity trend.

\section{S8 Reversibility}

In order to investigate the reversibility of the change with temperature, we have cycled the temperature between $30-60^{\circ} \mathrm{C}$, with one cycle of the data displayed in Fig. S7. We see that the same SFG signal was observed after heating and cooling. This indicates that dissolution of silica is an insignificant contribution to the surface environment during this short time frame. 


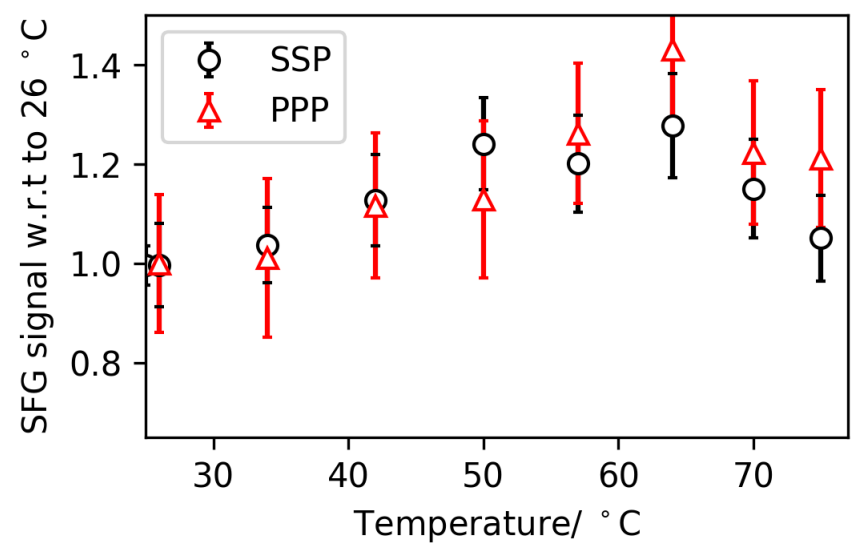

Figure S6: SSP (circles) and PPP (triangles) intensity at $3180 \mathrm{~cm}^{-1}$ plotted together as a function of temperature.

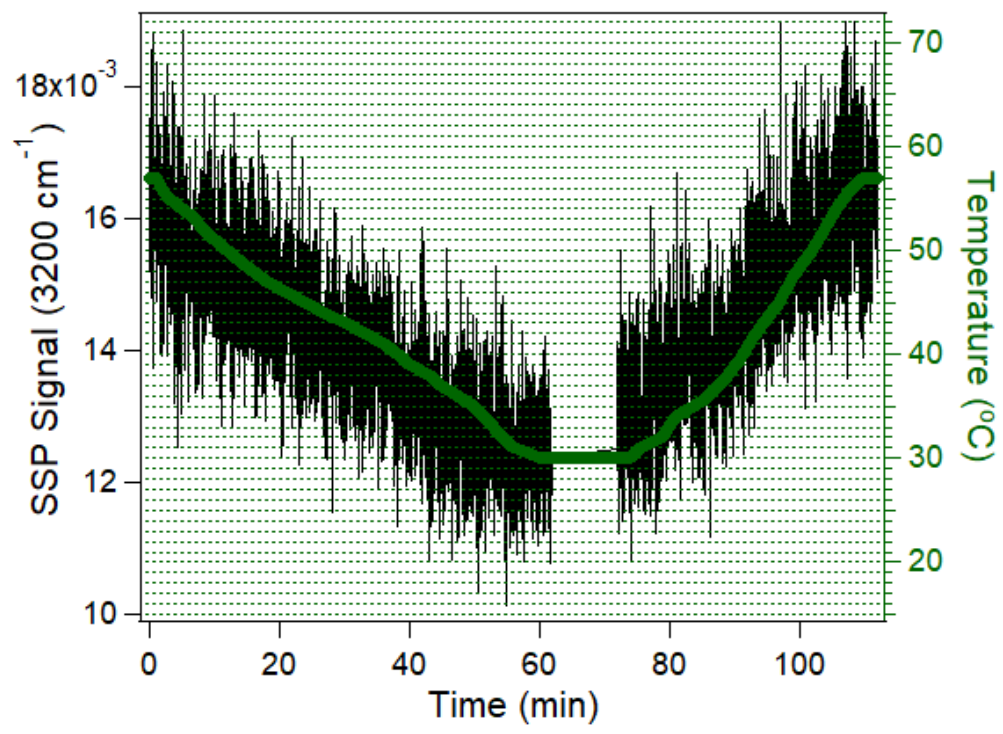

Figure S7: One cycle of a temperature ramp experiment in which the temperature was cycled between $30-60^{\circ} \mathrm{C}$. The SFG signal is plotted with respect to time on the left axis, and the corresponding temperature is shown on the right axis. 


\section{References}

[1] Jena, K. C.; Hore, D. K. J. Phys. Chem. C 2009, 113, 15364-15372.

[2] Ong, S.; Zhao, X.; Eisenthal, K. B. Chem. Phys. Lett. 1992, 191, 327-335.

[3] Wen, Y.-C.; Zha, S.; Liu, X.; Yang, S.; Guo, P.; Shi, G.; Fang, H.; Shen, Y. R.; Tian, C. Phys. Rev. Lett. 2016, 116, 016101.

[4] Gonella, G.; Lütgebaucks, C.; de Beer, A. G. F.; Roke, S. J. Phys. Chem. C 2016, 120, 9165-9173.

[5] Hore, D. K.; Tyrode, E. J. Phys. Chem. C 2019, 123, 16911-16920.

[6] Schaefer, J.; Gonella, G.; Bonn, M.; Backus, E. H. G. Phys. Chem. Chem. Phys. 2017, 19, 16875-16880.

[7] Zhang, C.; Sprik, M. Phy. Rev. B 2016, 93, 144201.

[8] Joutsuka, T.; Morita, A. J. Phys. Chem. C 2018, 122, 11407-11413.

[9] Bashkatov, A. N.; Genina, E. A. Proc. SPIE 2003, 5068, 393-395.

[10] Matsuoka, J.; Kitamura, N.; Fujinaga, S.; Kitaoka, T.; Yamashita, H. J. Non-Cryst. Solids 1991, 135, 86-89.

[11] Sulpizi, M.; Gaigeot, M.-P.; Sprik, M. J. Chem. Theory Comput. 2012, 8, 1037-1047.

[12] Light, T. S. Anal. Chem. 1984, 56, 1138-1142.

[13] Azam, M. S.; Darlington, A.; Gibbs-Davis, J. M. J. Phys.: Condens. Matter 2014, 26, 244107.

[14] Darlington, A. M.; Gibbs-Davis, J. M. J. Phys. Chem. C 2015, 119, 16560-16567.

[15] Zhuravlev, L. T. Coll. Surf. A: Phys. Eng. Asp. 2000, 173, 1-38.

[16] Brown, M. A.; Goel, A.; Abbas, Z. Angew. Chem. Int. Ed. 55, 2016, 3790-3794.

[17] Nagata, Y.; Hasegawa, T.; Backus, E. H. G.; Usui, K.; Yoshimune, S.; Ohto, T.; Bonn, M. Phys. Chem. Chem. Phys. 2015, 17, 23559-23564. 